Universidad Católica del Norte

Antofagasta - Chile

\title{
UNE INÉGALITÉ OPTIMALE POUR DES SOUS-VARIÉTÉS RIEMANNIENNES DANS DES ESPACES PSEUDO-RIEMANNIENS
}

\author{
B. Gmira \\ Université Abdelmalek Essaâdi, Katholieke Universiteit Leuven \\ and \\ L. Verstraelen \\ Université de Valenciennes Katholieke Universiteit Brussel
}

\begin{abstract}
Let $M^{n}$ be a Riemannian $n$-submanifold of a real space form $Q_{2}^{n+2}(c)$ of constant curvature $c$ and of index 2 . We prove a pontuise inequality relating the main Riemannian scalar invariants of $M^{n}$, namely the scalar curvature of $M^{n}$, the normal scalar curvature and the squared norm of the mean curvature vector of $M^{n}$ in $Q_{2}^{n+2}(c)$. Then we give some examples proving that the equality sign is in fact realized.
\end{abstract}

Key words: sous-variété riemannienne, courbure, espace à courbure constante.

AMS subject classifications: $53 \mathrm{B25}, 53 \mathrm{A35}, 53 \mathrm{A07}$ 


\section{Introduction}

Les deux conditions bien connues nécessaires à l'existence d'une immersion isométrique minimale d'une variété riemannienne $M^{n}$ dans l'espace euclidien, sont la non-compacité de $M$ et la non-positivité du tenseur de Ricci. Dans [4], S.S. Chern posait le problème de trouver d'autres telles conditions. Etant donnée une sous-variété riemannienne $M^{n}$ d'une variété réelle $N^{m}(c)$ à courbure constante $c$, on a comme conséquence directe de l'équation de Gauss, l'inégalité optimale suivante :

$$
\|H\|^{2} \geq \tau-c,
$$

qui relie en plus de $c$ la courbure scalaire normalisée $\tau$, et le carré de la norme du vecteur de courbure moyenne de $M^{n}$ dans $N^{m}(c)$. B-Y.Chen [3] a caractérisé les sous-variétés qui réalisent l'égalité dans $(0.1)$

En ce qui concerne des inégalités universelles, i.e. valables sans aucune condition sur les sous-variétés considérées, entre courbures extrinsèques et intrinsèques, B-Y.Chen [2] a obtenu récemment l'inégalité suivante:

$$
\delta_{M} \leq \frac{n^{2}(n-2)}{2(n-1)}\|H\|^{2}+\frac{1}{2}(n+1)(n-2) c,
$$

où $\delta_{M}=\frac{n(n-1)}{2} \tau(p)-\inf K(p)$, et $K(p)$ est la fonction courbure sectionnelle de $M^{n}$. $\Pi$ a également donné une caractérisation, en termes de la seconde forme fondamentale, du cas où l'égalité est réalisée dans (0.2). En particulier, cette inégalité donne une troisième condition nécessaire à l'existence d'une immersion isométrique minimale d'une variété riemannienne $M^{n}$ dans l'espace euclidien: les courbures sectionnelles et la courbure scalaire doivent satisfaire $K(\pi) \geq \frac{1}{2} \rho(p)$ pour toute section plane $\pi \subset T_{p} M, p \in M$.

Plus récemment encore, P.J. De Smet, F. Dillen, L. Verstraelen et L. Vrancken [5] ont démontré une nouvelle inégalité ponctuelle, pour toute sous-variété $M^{n}$ d'une variété $N^{n+2}(c)$ réelle à courbure constante $c$, faisant apparaitre en plus des invariants $\tau$ et $\|H\|^{2}$, un invariant extrinsèque additionnel qui est la fonction courbure normale $\tau^{N}$. Cette inégalité s'écrit:

$$
\|H\|^{2} \geq \tau+\tau^{N}-c .
$$

Ces mêmes auteurs ont donné dans leur article, des exemples de plusieurs classes de sous-variétés réalisant l'égalité dans (0.3), et ont conjecturé que cette inégalité est vraie en toutes codimensions. Cette conjecture est déja prouvée pour $n=2, c=0$ en codimension 2 par Wintgen [10]; pour $n=2$ 
en codimension quelconque par Guadalupe et Rodriguez [6]. Dans les deux cas, l'égalité est satisfaite en un point $p \in M$ si et seulement si l'ellipse de courbure en ce point est un cercle. Notons qu'en cas de connexion normale triviale, l'inégalité (0.3) se réduit à (0.1).

Si $Q_{2}^{n+2}(c)$ désigne une $(n+2)$-variété pseudo-riemannienne de signature $(2, n)$, à courbure constante $c$, nous obtenons alors le théorème suivant, qui est une version pseudo-riemannienne de l'inégalité (0.3).

Théorème Soit $M^{n} \longrightarrow Q_{2}^{n+2}(c)$ une immersion isométrique d'une $n$ variété riemannienne $M$ dans $Q_{2}^{n+2}(c)$. Alors en tout point de $M$, on a:

$$
-\|H\|^{2} \leq \tau-\tau^{N}-c .
$$

De plus, l'égalité est réalisée en un point si et seulement si on peut trouver une base orthonormée $\left(e_{1}, \ldots, e_{n}\right)$ de l'espace tangent et une base orthonormée $\left(\xi_{1}, \xi_{2}\right)$ de l'espace normal tel que les applications de Weingarten (cf. la formule de Weingarten, dans la section 1) s'écrivent sous la forme:

$$
\begin{aligned}
A_{\xi_{1}} & =(\lambda) \mu 0 \ldots 0 \mu \lambda 0 \ldots 000 \lambda \ldots 0 \ldots \ldots \ldots 000 \ldots \lambda \\
A_{\xi_{2}} & =(\mu) 00 \ldots 00-\mu 0 \ldots 0000 \ldots 0 \ldots \ldots \ldots .000 \ldots 0
\end{aligned}
$$

Nous retrouvons aussi certains exemples de classes de variétés, qui réalisent l'égalité dans (0.4), analogues à celles obtenues dans [5]. Dans la dernière section, nous montrons que l'égalité dans (0.4) est effectivement atteinte; notamment par certaines surfaces qui sont une version hyperbolique des surfaces de Vränceanu [Y].

\section{Préliminaires}

Soit $M^{n}$ une sous - variété riemannienne d'une variété pseudo -

riemannienne $Q_{n}^{m}(c)$ à courbure constante $c$, de signature $(m-n, n)$. Notons $\bar{\nabla}$, respectivement $\nabla$, la connexion de Levi- Civita associée à la métrique pseudo-riemannienne sur $Q_{n}^{m}(c)$, respectivement la connexion correspondant à la métrique induite sur $M$. La seconde forme fondamentale $h$ de $M$ dans $Q_{n}^{m}(c)$ est définie par la formule de Gauss:

$$
\bar{\nabla}_{X} Y=\nabla_{X} Y+h(X, Y),
$$


où $X, Y$ sont des champs de vecteurs tangents à $M . h(X, Y)$ est un champ de vecteurs normal à $M$ qui est symétrique en $X$ et $Y$. Soit $\xi$ un champ de vecteurs normal à $M$. Alors l'application de Weingarten $A_{\xi}$ relative $\dot{a} \xi$ et à la connexion normale $\nabla^{N}$ de $M$ dans $Q_{n}^{m}(c)$ est définie par la formule de Weingarten:

$$
\bar{\nabla}_{X} \xi=-A_{\xi} X+\nabla_{X}^{N} \xi
$$

où $-A_{\xi} X$ et $\nabla_{X}^{N} \xi$ sont respectivement la composante tangentielle et la composante normale de $\bar{\nabla}_{X} \xi$.

On prend un champ local de repères orthonormés $\left(e_{1}, \ldots, e_{n}, \xi_{1}, \ldots, \xi_{m-n}\right)$ sur $Q_{n}^{m}(c)$ tel que $\left(e_{1}, \ldots, e_{n}\right)$ sont tangents à $M$, et donc $\left(\xi_{1}, \ldots, \xi_{m-n}\right)$ sont normaux à $M$. Si $\left(\omega_{1}, \ldots, \omega_{m}\right)$ est le corepère associé au repère précédent, la métrique pseudo-riemannienne sur $Q_{n}^{m}(c)$ et la seconde forme fondamentale s'écrivent respectivement:

$$
\begin{gathered}
d s^{2}=\sum_{i=1}^{n} \omega_{i}^{2}-\sum_{\alpha=n+1}^{m} \omega_{\alpha}^{2}, \\
h=\sum_{\substack{1 \leq \alpha \leq m-n \\
1 \leq i, j \leq n}} \varepsilon_{\alpha} h_{i j}^{\alpha} \omega_{i} \otimes \omega_{j} \otimes \xi_{\alpha},
\end{gathered}
$$

où $h_{i j}^{\alpha}$ est un tenseur symétrique, $\varepsilon_{\alpha}=\left\langle\xi_{\alpha}, \xi_{\alpha}\right\rangle$ et $\langle,>$ désigne le produit indéfini associé à la métrique $\operatorname{sur} Q_{n}^{m}(c)$.

Les formes de connexion s' expriment dans le corepère tangent $\left(\omega_{1}, \ldots, \omega_{n}\right)$ :

$$
\omega_{i, \alpha+n}=\sum_{j=1}^{n} h_{i j}^{\alpha} \omega_{j}, \quad i=1, \ldots, n \text { et } \alpha=1, \ldots, m-n .
$$

Le vecteur de courbure moyenne défini par $H=\frac{1}{n}$ traceh s'écrit:

$$
H=\frac{1}{n} \sum_{i=1}^{n} h\left(e_{i}, e_{i}\right)=\frac{1}{n} \sum_{i=1}^{n} \sum_{\alpha=1}^{(m-n)} \varepsilon_{\alpha} h_{i i}^{\alpha} \xi_{\alpha},
$$

Soient $R$ et $R^{N}$ les tenseurs de courbure associés $\dot{a} \nabla$ et $\nabla^{N}$ respectivement. L'équation de Ricci s'écrit:

$$
R^{N}(X, Y) \xi=h\left(X, A_{\xi} Y\right)-h\left(A_{\xi} X, Y\right) .
$$

Rappelons la définition de la courbure scalaire normalisée $\tau$ définie dans [3], et celle de la courbure normale scalaire normalisée $\tau^{N}$ introduite dans [5]: 


$$
\tau=\frac{2}{n(n-1)} \sum_{1 \leq i<j \leq n}<R\left(e_{i}, e_{j}\right) e_{j}, e_{i}>
$$

$$
\tau^{N}=\frac{2}{n(n-1)} \sqrt{\sum_{1 \leq i<j \leq n} \sum_{1=\alpha<\beta}^{m-n}<R^{N}\left(e_{i}, e_{j}\right) \xi_{\alpha}, \xi_{\beta}>^{2}}
$$

Remarque Pour $n=2, \tau$ n'est autre que la courbure de Gauss $K$ de la surface $M$ et $\tau^{N}=\left|K^{N}\right|$, où $K^{N}$ est la courbure normale étudiée par Wintgen [10] (pour $m=4$ ), puis par Guadalupe et Rodriguez [6] pour $m$ quelconque.

\section{Démonstration du Théorème}

Dans cette section, on prend $m=n+2$, i.e. la sous-variété est de codimension 2. Les formules (1.2) et (1.4) se réduisent alors à:

$$
\begin{gathered}
H=-\frac{1}{n}\left\{\left(\sum_{i=1}^{n} h_{i i}^{1}\right) \xi_{1}+\left(\sum_{i=1}^{n} h_{i i}^{2}\right) \xi_{2}\right\} \\
\tau^{N}=\frac{2}{n(n-1)} \sqrt{\sum_{1 \leq i<j \leq n}<R^{N}\left(e_{i}, e_{j}\right) \xi_{1}, \xi_{2}>^{2}} .
\end{gathered}
$$

Pour démontrer le théorème $A$, nous aurons besoin de deux lemmes techniques et des deux inégalités triviales qui suivent:

$$
(a+b)^{2} \geq 4 a b,
$$

et l'égalité est réalisée dans (2.3) si et seulement si $a=b$.

$$
(A-C)^{2} \leq 2\left((A-B)^{2}+(B-C)^{2}\right),
$$

et l'égalité est réalisée dans (2.4) si et seulement si $B=\frac{1}{2}(A+C)$.

2.1. Lemme Soient $n$ nombres réels $a_{1}, a_{2}, \ldots, a_{n}$. On a:

$$
\sum_{i=1}^{n} a_{i}^{2}=\frac{1}{n-1}\left[\sum_{i<j}\left(a_{i}-a_{j}\right)^{2}+2 \sum_{i<j} a_{i} a_{j}\right] .
$$

2.2. Lemme[5] Soient n nombres réels $a_{1}, a_{2}, \ldots, a_{n}$, et soit $A=\sum_{i<j}\left(a_{i}-\right.$ $\left.a_{j}\right)^{2}$. On $a$ : 
$A \geq \frac{n}{2}\left(a_{1}-a_{2}\right)^{2}$ et l'égalité est satisfaite si et seulemen si $\frac{1}{2}\left(a_{1}+a_{2}\right)=$ $a_{3}=a_{4}=\ldots=a_{n}$.

Soient $k, l$ deus entiers tels que $1 \leq k<l \leq n$ et $(k, l) \neq(1,2)$. Si

$$
A=\frac{n}{2}\left(a_{1}-a_{2}\right)^{2}=\frac{n}{2}\left(a_{k}-a_{l}\right)^{2},
$$

alors $a_{1}=a_{2}=a_{3}=\ldots=a_{n}$.

Démonstration Pour $n=2$, le lemme est trivial. Supposons que le lemme est vrai pour un entier $n \geq 2$. Alors en appliquant successivement l'hypothèse de récurrence puis (2.4), on obtient:

$$
\begin{gathered}
\sum_{1 \leq i<j \leq n+1}\left(a_{i}-a_{j}\right)^{2} \geq \frac{n}{2}\left(a_{1}-a_{2}\right)^{2}+\left(a_{1}-a_{n+1}\right)^{2}+\left(a_{2}-a_{n+1}\right)^{2} \geq \\
\geq \frac{n+1}{2}\left(a_{1}-a_{2}\right)^{2},
\end{gathered}
$$

de plus, si l'égalité est satisfaite alor's $a_{3}=a_{4}=\ldots=a_{n}=a_{n+1}$ et $a_{n+1}=\frac{1}{2}\left(a_{1}+a_{2}\right)$. On voit immédiatement, par simple calcul que la réciproque est vraie.

Pour démontrer (2), on remarque que si $k, l>2$ alors d'après (1), $a_{k}=a_{l}$, donc $A=0$ et (2) découle immédiatement dans ce cas. On peut donc supposer que $l>2$ et que $k=2$. Appliquant alors (1) aux couples $(1,2)$ et $(2, l)$, on trouve que $a_{l}=\frac{1}{2}\left(a_{1}+a_{2}\right)$ et $a_{1}=\frac{1}{2}\left(a_{l}+a_{2}\right)$, d'où $a_{l}=a_{1}=a_{2}$, par conséquent $A=0$.

Nous sommes à présent en mesure de démontrer le Théorème $\mathrm{A}$.

Démonstration Soit $p \in M^{n}$. On choisit $\xi_{1}$ dans la direction du vecteur de courbure moyenne et $\xi_{2}$ un vecteur orthogonal. Ensuite, on prend une base orthonormée $\left(e_{1}, \ldots, e_{n}\right)$ telle que l'application $A_{\xi_{2}}$ soit un endomorphisme diagonal. L'équation de Ricci nous donne alors:

$$
<R^{N}\left(e_{i}, e_{j}\right) \xi_{2}, \xi_{1}>=<\left(h_{j j}^{2} h\left(e_{i}, e_{j}\right)-h_{i i}^{2} h\left(e_{i}, e_{j}\right)\right), \xi_{1}>=\left(h_{j j}^{2}-h_{i i}^{2}\right) h_{i j}^{1} .
$$

D'où:

$$
\frac{n(n-1)}{2} \tau^{N}=\sqrt{\sum_{1 \leq i<j \leq n}\left(h_{j j}^{2}-h_{i i}^{2}\right)^{2}\left(h_{i j}^{1}\right)^{2}} .
$$

L'équation de Gauss, traduite en termes de tenseurs de courbure donne:

$$
<R\left(e_{i}, e_{j}\right) e_{j}, e_{i}>=c\left(<e_{j}, e_{j}><e_{i}, e_{i}>-<e_{i}, e_{j}><e_{j}, e_{i}>\right)+
$$


$+i \mathrm{~h}\left(\mathrm{e}_{j}, e_{j}\right), h\left(e_{i}, e_{i}\right)>-<h\left(e_{i}, e_{j}\right), h\left(e_{j}, e_{i}\right)>=$

$=c+<h_{j j}, h_{i i}>-<h_{i j}, h_{i j}>$.

D'où:

$$
\frac{n(n-1)}{2}(\tau-c)=\sum_{1 \leq i<j \leq n}\left(<h_{i i}, h_{j j}>-<h_{i j}, h_{i j}>\right) .
$$

Maintenant, d'après (2.1) on a:

$$
\begin{gathered}
n^{2}\|H\|^{2}=\left(\sum_{i=1}^{n} h_{i i}^{1}\right)^{2}+\left(\sum_{i=1}^{n} h_{i i}^{2}\right)^{2}=\left(\sum_{i=1}^{n}\left(h_{i i}^{1}\right)^{2}+2 \sum_{i<j} h_{i i}^{1} h_{j j}^{1}\right)+ \\
\left(\sum_{i=1}^{n}\left(h_{i i}^{2}\right)^{2}+2 \sum_{i<j} h_{i i}^{2} h_{j j}^{2}\right) .
\end{gathered}
$$

En appliquant le lemme 2.1, on obtient:

$$
\begin{gathered}
n^{2}\|H\|^{2}=\frac{1}{n-1}\left(\sum_{i<j}\left(h_{i i}^{1}-h_{j j}^{1}\right)^{2}+\sum_{i<j}\left(h_{i i}^{2}-h_{j j}^{2}\right)^{2}\right)+ \\
\frac{2 n}{n-1} \sum_{i<j}\left(h_{i i}^{1} h_{j j}^{1}+h_{i i}^{2} h_{j j}^{2}\right)=\frac{1}{n-1}\left(\sum_{i<j}\left(h_{i i}^{1}-h_{j j}^{1}\right)^{2}+\sum_{i<j}\left(h_{i i}^{2}-h_{j j}^{2}\right)^{2}\right)- \\
-\frac{2 n}{n-1} \sum_{i<j}<h_{i i}, h_{j j}>,
\end{gathered}
$$

ce qui, d'après (2.5), devient:

$$
\begin{aligned}
n^{2}\|H\|^{2}= & \frac{1}{n-1}\left(\sum_{i<j}\left(h_{i i}^{1}-h_{j j}^{1}\right)^{2}+\sum_{i<j}\left(h_{i i}^{2}-h_{j j}^{2}\right)^{2}\right)-\frac{2 n}{n-1} \\
& \left(\frac{n(n-1)}{2}(\tau-c)+\sum_{i<j}<h_{i j}, h_{i j}>.\right.
\end{aligned}
$$

Puisque $A_{\xi_{2}}$ est diagonal, cette dernière égalité est équivalente à la suivante: 
$n^{2}(n-1)\left(\|H\|^{2}+\tau-c\right)=\left(\sum_{i<j}\left(h_{i i}^{1}-h_{j j}^{1}\right)^{2}+\sum_{i<j}\left(h_{i i}^{2}-h_{j j}^{2}\right)^{2}\right)+2 n \sum_{i<j}\left(h_{i j}^{1}\right)^{2}$

d'où:

$$
n^{2}(n-1)\left(\|H\|^{2}+\tau-c\right) \geq \sum_{i<j}\left(h_{i i}^{2}-h_{j j}^{2}\right)^{2}+2 n \sum_{i<j}\left(h_{i j}^{1}\right)^{2} .
$$

Ensuite, utilisant successivement (2.3) et le lemme 2.2, on aboutit enfin à :

$$
\begin{gathered}
\sum_{i<j}\left(h_{i i}^{2}-h_{j j}^{2}\right)^{2}+2 n \sum_{i<j}\left(h_{i j}^{1}\right)^{2} \geq \sqrt{8 n\left(\sum_{i<j}\left(h_{i i}^{2}-h_{j j}^{2}\right)^{2}\right)\left(\sum_{k<l}\left(h_{k l}^{1}\right)^{2}\right)} \\
\geq 2 n \sqrt{\sum_{k<l}\left(h_{k k}^{2}-h_{l l}^{2}\right)^{2}\left(h_{k l}^{1}\right)^{2}} \geq n^{2}(n-1) \tau^{N}
\end{gathered}
$$

d'où l'inégalité (0.4).

Supposons maintenant, qu'en un point $p$ l'égalité est atteinte dans (0.4). Dans ce cas, toutes les inégalités obtenues précédemment deviennent des égalités. Alors, en appliquant $(2.3)$ et le lemme 2.2. on trouve pour $(k, l)$, $1 \leq k<l \leq n$

$$
\begin{gathered}
h_{k k}^{1}-h_{l l}^{1}=0 \\
\operatorname{sum}_{i<j}\left(h_{i i}^{2}-h_{j j}^{2}\right)^{2}=2 n \sum_{i<j}\left(h_{i j}^{1}\right)^{2}, \\
h_{k l}^{1}=0 \text { ou } \frac{1}{2}\left(h_{k k}^{2}+h_{l l}^{2}\right)=h_{m m}^{2} \text { pour tout } m \neq k, l .
\end{gathered}
$$

Supposons d'abord que la seconde partie de (2.8) est vérifiée pour deux couples $(k, l)$. Alors, le lemme 2.2. implique que $h_{11}^{2}=h_{22}^{2}=\ldots=h_{n n}^{2}$. Puisque $\xi_{2}$ est orthogonal à $H$, on a $h_{i i}^{2}=0$ pour tout $i$, et (2.7) donne $h_{i j}^{1}=0$ pour $i \neq j$ donc $M^{n}$ est totalement ombilicale d'après (2.6).

Supposons maintenant que la seconde partie de (2.8) est vérifiée pour un seul couple $(k, l)$. On peut supposer $k=1$ et $l=2$, quitte à réordonner la base $\left(\epsilon_{1}, \ldots, e_{n}\right)$. Ensuite, en posant $h_{11}^{2}=\mu$ et $h_{22}^{2}=\nu$, on a $h_{m m}^{2}=$ 
$\frac{1}{2}(\mu+\nu)$, d'où $\nu=-\mu$ puisque $\xi_{2}$ est orthogonal à $H$. D'après notre hypothèse, $(2.8)$ donne $h_{k l}^{1}=0$ pour $k<l,(k, l) \neq(1,2)$. Et, $(2.7)$ se réduit à:

$$
2 n\left(h_{12}^{1}\right)^{2}=4 \mu^{2}+2(n-2) \mu^{2}=2 n \mu^{2} .
$$

Ainsi, avec (2.6), et quitte à changer $e_{2}$ en $-e_{2}$ si nécessaire, la deuxième partie du théorème est démontrée dans ce cas.

Enfin, si la seconde partie de (2.8) n'est jamais satisfaite, alors $h_{k l}^{1}=0$ pour tous les indices $k<l$. Dans ce cas, $(2.7)$ entrainerait $h_{11}^{2}=\ldots=h_{n n}^{2}$, ce qui contredit l'hypothèse.

\section{Quelques classes de sous-variétés réalisant l'égalité}

3.1. Les sous-variétés totalement ombilicales Ce sont les sous variétés pour lesquelles les opérateurs de Weingarten sont proportionnels à l'identité. Les sous-variétés totalement ombilicales de dimension $n$, d'une $(n+2)$ variété pseudo-riemannienne de signature $(2, n)$ à courbure constante satisfont clairement l'égalité dans (0.4). Une classification des sous-variétés totalement ombilicales d'une variété à courbure constante est donnée dans [1].

3.2. Les surfaces dont l'ellipse de courbure est un cercle Pour davantage de détails sur la notion d'ellipse de courbure, voir [8] ou [11]. C'est le sousespace de l'espace normal défini par $\left\{h(X, X): X \in T_{p} M,\|X\|=1\right\}$, où $h$ est la seconde forme fondamentale de l'immersion et || || la norme des vecteurs. Les surfaces d'une variété $Q_{2}^{4}(c)$ pour lesquelles l'ellipse de courbure est un cercle réalisent l'égalité dans (0.4) [7]. Un cas particulier de cette classe de surfaces est constitué par les surfaces superminimales (i.e. minimales ayant une ellipse de courbure circulaire) de l'espace pseudoeuclidien $E_{2}^{4}$. Les cylindres, au-dessus de telles surfaces réalisent également l'égalité.

3.3. Les courbes planes holomorphes Les courbes holomorphes dans $C_{1}^{2}$ sont des surfaces superminimales de $E_{2}^{4}\left(C_{1}^{2}\right.$ étant l'espace pseudo-euclidien complexe de dimension 2 et de signature $(2,2)$ ). Par conséquent les cylindres complexes, au-dessus de courbes holomorphes de $C_{1}^{2}$, satisfont aussi l'égalité dans (0.4). 
3.4. Produit tordu (warped product) [9] Soient $\left(M_{1}, g_{1}\right)$ et $\left(M_{2}, g_{2}\right)$ deux variétés riemanniennes, et $\rho$ une fonction positive $C^{\infty}$ sur $M_{1}$. Le produit tordu (warped product) $M_{1} \times_{\rho} M_{2}$ de $\left(M_{1}, g_{1}\right)$ et $\left(M_{2}, g_{2}\right)$ est la variété produit $M_{1} \times M_{2}$ munie de la métrique $g=g_{1} \times_{\rho} g_{2}$ définie par:

$$
g_{1} \times_{\rho} g_{2}=\pi_{1}^{*} g_{1}+\left(\rho \circ \pi_{1}\right) \pi_{2}^{*} g_{2},
$$

$\pi_{i}: M_{1} \times M_{2} \longrightarrow M_{i}$ étant les projections naturelles, $i=1,2$. On obtient alors une série d'exemples en décomposant l'espace $E_{2}^{n+2}$ en produit tordu $E_{2}^{n+2}=E^{n-2} \times_{\rho} E_{2}^{4}$. Ainsi, prenant une surface superminimale $M^{2}$ de $E_{2}^{4}$ et formant la sous-variété $E^{n-2} \times_{\rho} M^{2}$ de $E_{2}^{n+2}$, on obtient encore une sous-variété qui réalise l'égalité dans (0.4).

\section{Version hyperbolique des surfaces de Vrănceanu}

Dans l'espace pseudo-euclidien $E_{2}^{4}$ muni de la métrique

$$
\bar{g}=d x_{1}^{2}+d x_{2}^{2}-d x_{3}^{2}-d x_{4}^{2},
$$

on considère les surfaces de rotation $M$ dont le vecteur position est donné par:

$$
X(u, v)=r(u)(\operatorname{ch} u \cdot \operatorname{sh} v, \operatorname{sh} u \cdot \operatorname{ch} v, \operatorname{sh} u \cdot \operatorname{sh} v, \operatorname{ch} u \cdot \operatorname{ch} v),
$$

où $(u, v) \in R^{2}$ et $r(u)$ est une fonction différentiable. La première forme fondamentale est décrite par:

$$
d s^{2}=\left(r^{2}-r^{\prime 2}\right) d u^{2}+r^{2} d v^{2} .
$$

On choisit un repère mobile $\left(e_{1}, e_{2}, e_{3}, e_{4}\right)$ de $E_{2}^{4}$ tel que $\left(e_{1}, e_{2}\right)$ soit tangent à $M$ et $\left(e_{3}, e_{4}\right)$ soit normal à $M$;

$$
\begin{gathered}
e_{1}=(\operatorname{ch} u \cdot \operatorname{ch} v, \operatorname{sh} u \cdot \operatorname{sh} v, \operatorname{sh} u \cdot \operatorname{ch} v, \operatorname{ch} u \cdot \operatorname{sh} v), \\
e_{2}=\frac{1}{A}(B \operatorname{sh} v, C \operatorname{ch} v, C \operatorname{sh} v, B \operatorname{ch} v), \\
e_{3}=\frac{1}{A}(C \operatorname{sh} v, B \operatorname{ch} v, B \operatorname{sh} v, C \operatorname{ch} v), \\
e_{4}=-\varepsilon(\operatorname{sh} u \cdot \operatorname{ch} v, \operatorname{ch} u \cdot \operatorname{sh} v, \operatorname{ch} u \cdot \operatorname{ch} v, \operatorname{sh} u \cdot \operatorname{sh} v),
\end{gathered}
$$


où

$$
\begin{gathered}
\left\{A=\sqrt{\varepsilon\left(r^{2}-r^{\prime 2}\right)}, \varepsilon= \pm 1 \text { de façon à ce que } \varepsilon\left(r^{2}-r^{\prime 2}\right)>0, B=\right. \\
=r^{\prime} \operatorname{ch} u+r \operatorname{sh} u \text { et } C=r^{\prime} \operatorname{sh} u+r \operatorname{ch} u .
\end{gathered}
$$

$\mathrm{Si}<,>$ désigne le produit scalaire associé à la métrique $\bar{g}$ sur $E_{2}^{4}$, on vérifie aisément que l' on a:

$$
<e_{1}, e_{1}>=-<e_{4}, e_{4}>=1,<e_{2}, e_{2}>=-<e_{3}, e_{3}>=\varepsilon .
$$

Le repère $\left(e_{1}, e_{2}\right)$ et le corepère associé s'écrivent en fonction de $u$ et $v$ comme suit:

$$
e_{1}=\frac{1}{r} \frac{\partial}{\partial v}, e_{2}=\frac{1}{\varepsilon A} \frac{\partial}{\partial u}, \omega_{1}=r d v \text { et } \omega_{2}=\varepsilon A d u .
$$

Les iormes locales de structure sont déterminées à partir des équations

$$
d e_{i}=\sum_{k} \varepsilon_{k} \omega_{i k} e_{k}, \quad \varepsilon_{k}=<e_{k}, e_{k}>
$$

On trouve:

$$
\begin{aligned}
& \left\{\omega_{12}=-\frac{r^{\prime}}{r A} \omega_{1}, \omega_{13}=-\frac{1}{A} \omega_{1}, \omega_{14}=\frac{1}{A} \omega_{2}, \omega_{23}=\right. \\
& \frac{\varepsilon}{A^{3}}\left(2 r^{\prime 2}-r r^{\prime \prime}-r^{2}\right) \omega_{2}, \quad \omega_{24}=\frac{1}{\varepsilon A} \omega_{1}, \omega_{34}=\frac{r^{\prime}}{\varepsilon r A} \omega_{1} .
\end{aligned}
$$

De ces équations et de la relation (1.1), on déduit la seconde forme fondamentale:

$\left\{h_{11}^{1}=-a, h_{12}^{1}=h_{21}^{1}=0, h_{22}^{1}=\varepsilon b, h_{11}^{2}=h_{22}^{2}=0, h_{12}^{2}=h_{21}^{2}=\varepsilon a\right.$, (4.1)

où l'on a posé:

$$
a=A^{-1} \text { et } b=\varepsilon A^{-1}\left(2 r^{\prime 2}-r r^{\prime \prime}-r^{2}\right) .
$$

Ceci nous permet de déterminer le vecteur de courbure moyenne, la courbure de Gauss $K$ et la courbure normale $K^{N}$ de la surface $M$ dans $E_{2}^{4}$. On obtient: 


$$
\begin{gathered}
H=\frac{1}{2} \varepsilon(a-b) e_{3}, \quad \tau=K=a b+a^{2}, \\
\tau^{N}=\left|K^{N}\right|=|a(a+\varepsilon b)| .
\end{gathered}
$$

Notons que lorsque $\varepsilon=+1$, i.e. dans le cas où $r^{2}-r^{\prime 2}>0$, la surface $M$ est une sous-variété riemannienne de l'espace pseudo-euclidien $E_{2}^{4}$; nous sommes donc dans les hypothèses du théorème $\mathrm{A}$ et il est aisé de vérifier l'inégalité (0.4) dans ce cas. Nous avons en effet:

$$
4\left(-\|H\|^{2}-\tau\right)=-\left[(a+b)^{2}+4 a^{2}\right] \leq-4|a(a+b)|, \leq-4 \tau^{N} .
$$

D'autre part, les opérateurs de Weingarten s'écrivent dans la base

$$
\left(e_{1}, e_{2}, \xi_{1}, \xi_{2}\right)
$$

où $\xi_{1}=e_{3}$ et $\xi_{2}=e_{4}$, sous la forme:

$$
A_{\xi_{1}}=(-) a 00 b, A_{\xi_{2}}=(0) a a 0
$$

On vérifie, à l'aide d'un calcul très simple d'algèbre linéaire, qu'il existe une base $\left(e_{1}^{\prime}, e_{2}^{\prime}\right)$ de l'espace tangent dans laquelle on a:

$$
\begin{gathered}
A_{\xi_{1}}=() b-a 2 \frac{\varepsilon_{1}(a+b)}{2 \varepsilon_{2}} \frac{\varepsilon_{1}(a+b)}{2 \varepsilon_{2}} \frac{b-a}{2}, \\
A_{\xi_{2}}=() \varepsilon_{1}(a+b) 2 \varepsilon_{2} 00-\frac{\varepsilon_{1}(a+b)}{2 \varepsilon_{2}},
\end{gathered}
$$

si et seulement si l'égalité est satisfaite dans (4.2). Cette base est donnée par:

$$
e_{1}^{\prime}=\alpha\left(e_{1}-\varepsilon_{1} \varepsilon_{2} e_{2}\right), e_{2}^{\prime}=\alpha\left(\varepsilon_{1} e_{1}+\varepsilon_{2} e_{2}\right) \text { avec } \varepsilon_{i}= \pm 1 \text { et } \alpha \in R^{*} .
$$

Remarque L'égalité est satisfaite dans (4.2) si et seulement si on a:

$$
|a+b|=2|a| .
$$

Et ceci équivaut, d'après (4.1), à $\left\|h_{11}-h_{22}\right\|=2\left\|h_{12}\right\|$; autrement dit l'ellipse de courbure est un cercle. En résolvant l'équation (4.3), on constate que l'égalité dans (4.2) est effectivement atteinte par les surfaces $M$ données par: 


$$
\begin{gathered}
\{X(u, v)=r(u)(\operatorname{ch} u \cdot \operatorname{sh} v, \operatorname{sh} u \cdot \operatorname{ch} v, \operatorname{sh} u \cdot \operatorname{sh} v, \operatorname{ch} u \cdot \operatorname{ch} v) r(u)= \\
k \operatorname{ch}(-2 u+l) \operatorname{our}(u)=k \operatorname{ch}(2 u+l),(u, v) \in R^{2},
\end{gathered}
$$

avec $k$ et $l$ des constantes arbitraires d'intégration. Parmi ces surfaces, les minimales sont celles pour lesquelles $r(u)=k \operatorname{ch}(-2 u+l)$.

\section{References}

[1] B. Y. Chen paper Geometry of submanifolds Marcel Dekker, New York, (1973).

[2] B. Y. Chen Some pinching and classification theorems for minimal submanifolds Archiv. Math., pages 568-578, Vol. 60, (1993).

[3] B. Y. Chen Mean curvature and shape operator of isometric immersions in real space-forms Glasgow Math. J, pages 87-97, Vol. 38, (1996).

[4] S. S. Chern Minimal submanifolds in a Riemannian manifold Univ. of Kansas, Lawrence, Kansas, (1968).

[5] P. J. De Smet, F. Dillen, L. Verstraelen and L. Vranken A pointwise inequality in Submanifold Theory, preprint.

[6] I. V. Guadalupe and L. Rodriguez Normal curvature of surfaces in space forms Pacific J. Math., pages 95-103, Vol. 106, (1983).

[7] B. Gmira et L. Verstraelen Les courbures externes et internes des surfaces riemanniennes dans des espaces pseudo-riemanniens, à paraître.

[8] J. A. Little paper On singularities of submanifolds of higher dimensional Euclidian space jour Ann. Mat. Pura Appl., pages 261-335, Vol. 83, (1996).

[9] B.O'Neill Semi-Riemannian Geometry with applications to Relativity Academic Press, Inc., (1983). 
[10] P. Wintgen Sur l'inégalité de Chen-Willmore C. R. Acad. Sc. Paris. pages 993-995, Vol. 288, (1979).

[11] Y. C. Wong Contributions to the theory of surfaces in 4-space of constant curvature Trans. Amer. Math. Soc., pages 467-507, Vol. 59, (1946),

Received : March 10, 1997.

\section{B. Gmira}

Université Abdelmalek Essaâdi

Faculté des Sciences

B. P.: 2121

Tétouan (Maroc)

Université de Valenciennes

Lamath Groep Exacte Wetenschappen

Le Mont Houy, BP 311

59304 Valenciennes Cedex (France)

\section{Verstraelen}

Katholieke Universiteit Leuven

Departement $\mathrm{W}$ iskunde

Celestijnenlaan 200 B

B 3001 Leuven (Belgium)

Katholieke Universiteit Brussel

Vrijheidslaan 17

B 1081 Brussel (Belgium) 